\title{
Induced reprogramming of adult murine cardiomyocytes to pluripotency in vivo
}

\author{
Irene de Lázaro ${ }^{1,2}$, Tiara L Orejón-Sánchez ${ }^{1,3}$, Christina M Tringides ${ }^{2,4,5}$ and \\ David J Mooney ${ }^{1,2 *}$ \\ 1 John A. Paulson School of Engineering and Applied Sciences, Harvard University, Cambridge, MA, USA \\ ${ }^{2}$ Wyss Institute for Biologically Inspired Engineering at Harvard University, Boston, MA, USA \\ ${ }^{3}$ University of Applied Sciences Technikum Wien, Vienna, Austria \\ ${ }^{4}$ Harvard Program in Biophysics, Harvard University, Cambridge, MA, USA \\ ${ }^{5}$ Harvard-MIT Division in Health Sciences and Technology, Massachusetts Institute of Technology, \\ Cambridge, MA, USA
}

\footnotetext{
* Correspondence should be addressed to: mooneyd@seas.harvard.edu
} 


\section{ABSTRACT}

Partial cell reprogramming has been demonstrated in certain mouse tissues by in situ overexpression of Oct3/4, KIf4, Sox2 and cMyc (OKSM) transcription factors, and can trigger rejuvenation and/or augment regeneration of aged or injured tissues. In vivo reprogramming of adult mouse cardiomyocytes has been elusive, but success could overcome the lack of endogenous cardiomyocyte turnover that contributes to the poor resolution of heart disease. Here, we exploited cell type-specific Cre recombination and conditional, doxycycline-inducible, control of gene expression to generate cardiomyocyte-specific, inducible, reprogrammable mice. Eighteen days of doxycycline-induced OKSM expression in this model established a gene expression program characteristic of the pluripotent state and triggered the generation of teratomas of confirmed cardiomyocyte origin. These findings confirm that OKSM reprograms adult mouse cardiomyocytes to pluripotency and will enable studies of the contribution of reprogrammed cardiomyocytes to cardiac regeneration.

Keywords: cardiomyocyte, de-differentiation, reprogramming, pluripotency 


\section{INTRODUCTION}

Expression of Oct3/4, KIf4, Sox2 and cMyc (OKSM) transcription factors in adult mouse tissues generates reprogrammed cells in situ (Abad et al., 2013; de Lazaro et al., 2019; Yilmazer et al., 2013). These commit to the pluripotent state and can form undifferentiated tumors, or teratomas, when OKSM expression is maintained (Abad et al., 2013). However, reprogramming is a stepwise process (Polo et al., 2012) and, with shorter OKSM expression, cells undergo partial reprogramming, a so far broadly-defined term that can range from epigenetic rejuvenation - the erasure of epigenetic marks associated with cellular ageing (Lu et al., 2020; Ocampo et al., 2016) - to transient and reversible loss of cell identity, accompanied by time-limited proliferation and reactivation of pluripotency-related genes (de Lazaro et al., 2019; Yilmazer et al., 2013). Partial in vivo cell reprogramming has been proposed as an approach to enhance the regenerative capabilities of adult tissues that could benefit from the lack of ex vivo manipulations, the versatility of the OKSM factors to enable reprogramming of a wide variety of cell types, and the typically enhanced cell maturation that is achieved in vivo versus in vitro (de Lazaro et al., 2017; de Lazaro and Kostarelos, 2014). To date, mouse preclinical studies have demonstrated that in vivo reprogramming through OKSM overexpression enhances regeneration of skeletal muscle (de Lazaro et al., 2019; Wang et al., 2021) and retina (Lu et al., 2020), improves wound healing (Doeser et al., 2018) and rejuvenates several tissues in progeroid and naturally aged mice (Lu et al., 2020; Ocampo et al., 2016). A recent study has also suggested that reprogramming early in life could have long-lasting effects, significantly increasing life-span and overall fitness at old age (Alle et al., 2021). Confirmation of the regenerative effects of in vivo reprogramming in multiple independent laboratories, in different tissues and disease models, supports the promise of this strategy.

Most studies have focused primarily on demonstrating regenerative or rejuvenating effects linked to in vivo cell reprogramming, but in many cases the starting cell type (or types) that 
undergo reprogramming have not been identified (Alle et al., 2021; de Lazaro et al., 2019; Doeser et al., 2018; Ocampo et al., 2016). Most studies are conducted using transgenic reprogrammable mice that exploit tetracycline-inducible systems to control OKSM expression (Abad et al., 2013; Alle et al., 2021; Doeser et al., 2018; Ocampo et al., 2016). While this strategy allows high levels of OKSM expression that ensure efficient reprogramming, and tight temporal control over reprogramming required to avoid tumorigenesis, OKSM expression is typically ubiquitous and thus does not enable cell-type specificity of reprogramming. Delivery of exogenous OKSM factors, with or without viral vectors, can offer tissue targeting capabilities - but typically not with cell type resolution - and is often limited by poor temporal control of OKSM expression (e.g. retroviral and AAV integrating vectors that trigger tumorigenesis due to extended OKSM expression (Senis et al., 2018; Seo et al., 2016)) and/or limited efficiency of reprogramming (e.g. plasmid DNA (de Lazaro et al., 2019) and adenoviral vectors (Kisby et al., 2021a)).

Given the negligible turnover of adult mammalian cardiomyocytes (Eschenhagen et al., 2017), in vivo partial reprogramming of this cell type could enable proliferation after cardiac injury and a new approach to achieve cardiac regeneration. However, the ability of terminally differentiated, adult cardiomyocytes to undergo OKSM-induced reprogramming has been unclear. A negative correlation between a cell's differentiation state and its capability to undergo OKSMtriggered reprogramming has been consistently described for various cell lineages (Eminli et al., 2009; Polo et al., 2010; Tan et al., 2011), which may explain why reprogramming of cardiac tissues has not been reported in reprogrammable mice with ubiquitous OKSM expression even after they developed lethal teratomas in other organs (mainly the pancreas and gut) (Abad et al., 2013). Adenoviral vectors have shown to induce transient reprogramming in neonatal rat and mouse cardiomyocytes in vitro (Kisby et al., 2021b), but the same effect has not been confirmed in adult counterparts in vivo (Kisby et al., 2021a).

In this work, we combined the temporal control over gene expression offered by a tet-ON tetracycline-inducible system with the power of Cre recombination under the control of a cell-type 
specific promoter to generate cardiomyocyte-specific, inducible, reprogrammable mice - herein also referred to as Myh6-Cre ${ }^{+} \mathrm{Col} 1 \mathrm{a} 1^{\mathrm{OKSM}}$ mice - and determine the ability of adult mammalian cardiomyocytes to undergo reprogramming. We demonstrate that OKSM expression is induced exclusively in cardiomyocytes by oral administration of doxycycline in this model, and that these cells undergo complete reprogramming to bona fide pluripotency in situ.

\section{RESULTS}

A cardiomyocyte-specific, inducible, reprogrammable mouse. To investigate if adult mouse cardiomyocytes can undergo OKSM-induced reprogramming in vivo, we generated triple mutant mice in which the expression of the four reprogramming factors is induced exclusively in cardiomyocytes and at times of choice (Figure 1a). In brief, cardiomyocyte-specific expression of Cre recombinase, driven by the cardiomyocyte-specific alpha myosin-heavy chain Myh6 promoter (Agah et al., 1997), was combined with a tet-ON doxycycline-inducible system to control the expression of OKSM (Stadtfeld et al., 2010) and conditional expression of the reverse tetracycline-controlled transactivator (rtTA) (Belteki et al., 2005). rtTA and GFP are preceded by a STOP sequence flanked by loxP sites - thus excised only in cardiomyocytes, by Cre - and, in the presence of doxycycline, rtTA induces OKSM expression. Fluorescence activated cell sorting (FACS) of different cardiac cell types (cardiomyocytes, cardiac fibroblasts, endothelial cells and leucocytes) demonstrated that GFP expression - and thus Cre recombination - is restricted to cardiomyocytes of Myh6-Cre ${ }^{+}$mice (Figure 1b-c). To obtain further proof of the cardiomyocyte specificity of the system, $1 \mathrm{mg} / \mathrm{ml}$ doxycycline was administered in the drinking water of Myh6$\mathrm{Cre}^{+} \mathrm{Col} 1 \mathrm{a} 1^{\mathrm{OKSM}}$ mice for a week and cardiomyocytes, cardiac fibroblasts and cardiac endothelial cells were isolated with a combination of centrifugation and magnetic cell sorting (Figure 1d). Real-time, reverse-transcription, quantitative PCR (RT-qPCR) analysis demonstrated that Oct3/4 
bioRxiv preprint doi: https://doi.org/10.1101/2021.12.22.473302; this version posted December 23, 2021. The copyright holder for this preprint

(which was not certified by peer review) is the author/funder, who has granted bioRxiv a license to display the preprint in perpetuity. It is made available under aCC-BY-NC-ND 4.0 International license.

and GFP were significantly expressed in cardiomyocytes only (Figure 1e-f). To confirm the purity of the different cell types isolated, the expression levels of cell-specific markers Myh/2, Ddr2 and Vwf - a cardiomyocyte, cardiac fibroblast and endothelial cell specific marker, respectively - were measured and found to be significantly upregulated in the respective cell fractions (Figure $\mathbf{1} \mathbf{g}-\mathbf{i}$ ). Analysis of other organs, including the liver (Figure 1j), spleen (Figure 1k) and kidney (Figure 1I) confirmed the absence of off-target expression of the GFP and OKSM cassettes. 
a
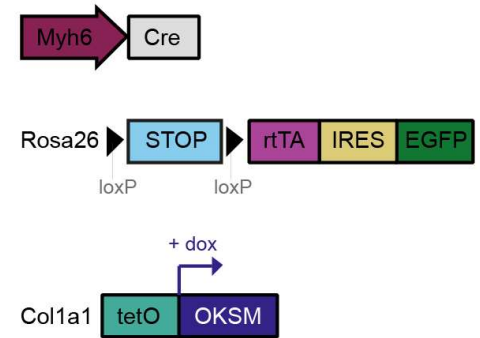

C

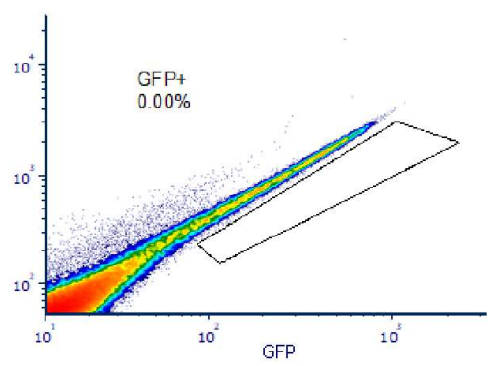

d

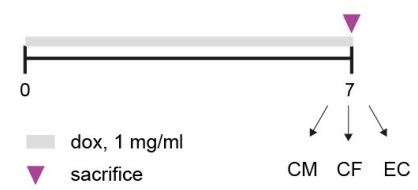

9
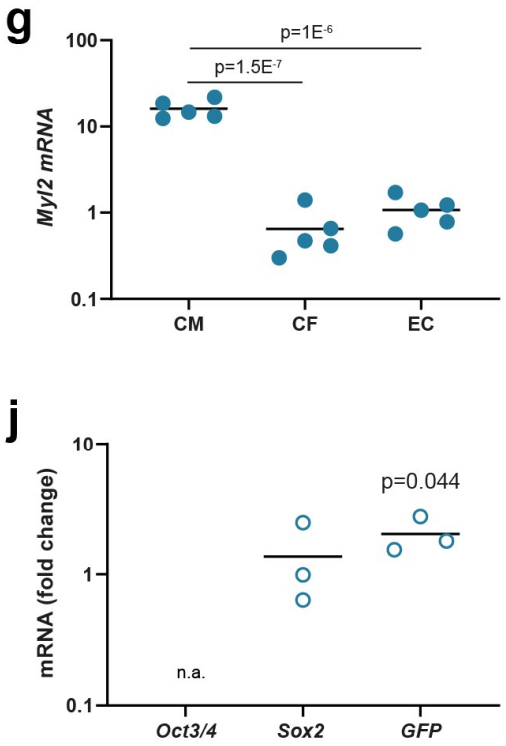

b

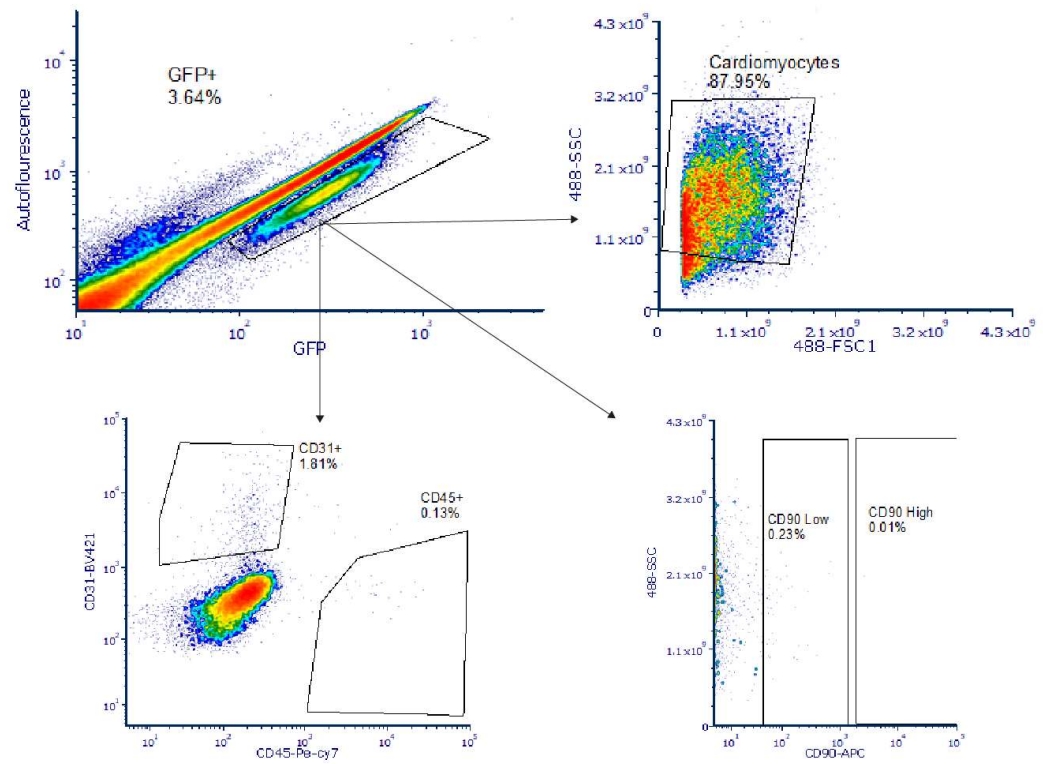

e

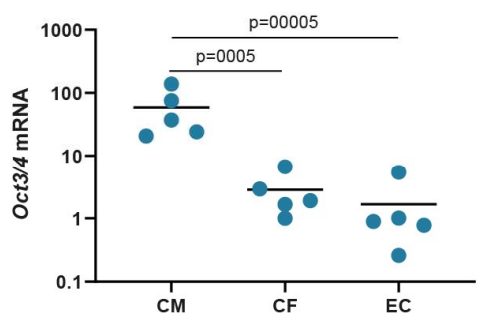

f

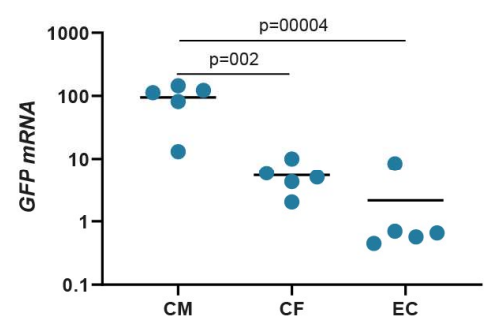

h

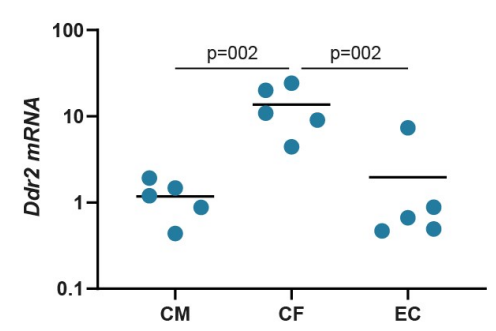

i

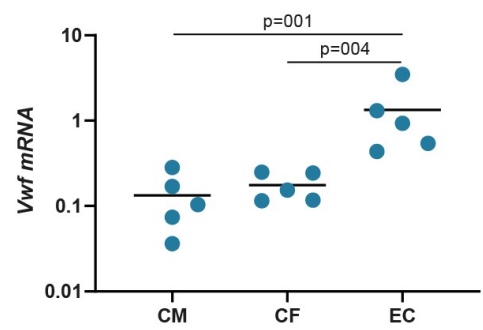

$\mathbf{k}$

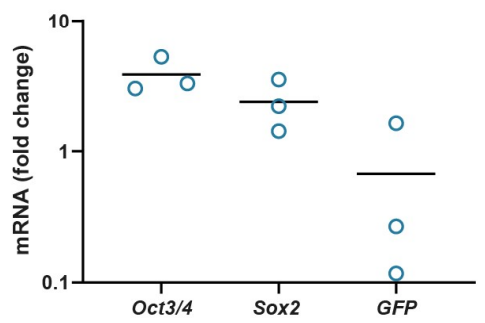

I

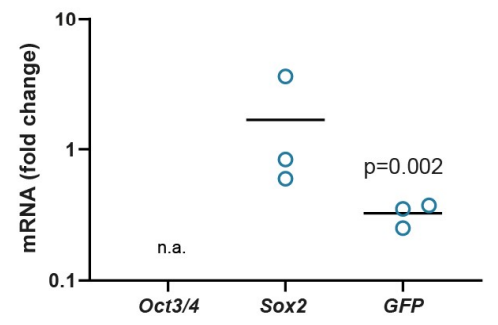

Figure 1. Generation and characterization of cardiomyocyte-specific, inducible, reprogrammable mice. (a) Schematic representation of the gene circuits utilized to create cardiomyocyte-specific, inducible, reprogrammable mice. (b) Representative FACS sorting plots of $\mathrm{GFP}^{+}$cells from Myh6-Cre ${ }^{+} \mathrm{Col} 1 \mathrm{a} 1{ }^{\mathrm{OKSM}}$ mice $(n=5)$, demonstrating isolation of cardiomyocytes (top right, sorted based on size), and depletion of endothelial cells and leucocytes (bottom left, 
sorted based on CD31 and CD45 positivity, respectively) and cardiac fibroblasts (bottom right, sorted based on CD90 expression). (c) Representative FACS sorting plot of $\mathrm{GFP}^{+}$cells from Myh6-Cre-Col1a1 ${ }^{\mathrm{OKSM}}$ mice $(\mathrm{n}=2)$. (d) Schematic representation of the experimental design to analyze gene expression in different cardiac cell types after doxycycline administration. mRNA levels of the reprogrammable factor Oct3/4 (e), GFP (f) and cell-type specific genes - Myl2, CMspecific (g); Ddr2, CF-specific (h) and Vwf, EC-specific (i) - in different cardiac cell types after 7 days of doxycycline administration. CM: cardiomyocyte, CF: cardiac fibroblast, EC: endothelial cells. Gene expression levels were normalized to those in cardiomyocytes. Individual data points represent the fold change $\left(2^{\wedge-\Delta \Delta C t}\right)$ of each replicate $(n=5$ mice/group). Statistical analysis was performed by one-way ANOVA and Tukey's post-hoc test. Gene expression in liver (f), spleen (g) and kidney (h) tissues of Myh6-Cre ${ }^{+} \mathrm{Col} 1 \mathrm{a}{ }^{\mathrm{OKSM}}$ mice. Relative expression was normalized to that of control Myh6-Cre ${ }^{+} \mathrm{Col} 1 \mathrm{a} 1^{\mathrm{OKSM}}$ mice. Individual data points represent the fold change $\left(2^{\wedge-\Delta \Delta \mathrm{Ct}}\right)$ of each replicate ( $n=3$ mice/group). $n$.a.: no amplification. Statistical analysis was performed by oneway ANOVA. $P$ values refer to statistical significance in relevance to the control group.

\section{OKSM expression establishes a genetic program characteristic of pluripotency and}

induces cardiomyocyte de-differentiation. The drinking water of reprogrammable (Myh6$\left.\mathrm{Cre}^{+} \mathrm{Col} 1 \mathrm{a} 1^{\mathrm{OKSM}}\right)$ or control $\left(\mathrm{Myh} 6-\mathrm{Cre}^{+} \mathrm{Col} 1 \mathrm{a} 1^{\mathrm{WT}}\right)$ mice was next supplemented with $1 \mathrm{mg} / \mathrm{ml}$ doxycycline for 7,12 and 18 days, and changes in gene expression in the ventricular myocardium analyzed (Figure 2a). Increased expression of OKSM factors was detected by day 7 in reprogrammable mice treated with doxycycline, and further increased on day 12 to reach a plateau (Figure 2b-c). On day 12, a slight, albeit not statistically significant, elevation was found in the mRNA levels of pluripotency-related genes, including Nanog, the endogenous form of Oct4 (endoOct4) and Gdf3 in the reprogrammable group (Figure 2d). These genes, which are typically silenced in adult differentiated cells but re-expressed from the maturation phase of iPSC reprogramming (David and Polo, 2014), were significantly and very highly upregulated on day 18 (>1000 fold, on average, for Nanog and >100 fold, on average, for endoOct4 and Gdf3). We confirmed that these changes in the expression of OKSM factors and endogenous pluripotency genes were only observed in triple-mutant, reprogrammable mice (Myh6-Cre ${ }^{+} \mathrm{Col} 1 \mathrm{a} 1{ }^{\mathrm{OKSM}}$ ) administered with doxycycline but not in mutants lacking Cre recombination (Myh6-CreCol1a1 $\left.{ }^{\mathrm{OKSM}}\right)$ or the OKSM cassette $\left(\mathrm{Myh6}-\mathrm{Cre}^{+} \mathrm{Col} 1 \mathrm{a} 1^{\mathrm{WT}}\right)$, or in reprogrammable mice that were administered unadulterated, normal drinking water (Figure S1). Raising the dose of doxycycline to $2 \mathrm{mg} / \mathrm{ml}$ did not trigger higher mRNA expression levels of the OKSM reprogramming factors 
(on day 18) or of the endogenous pluripotency genes investigated, at any of the time points analyzed (Figure S2). Therefore, the $1 \mathrm{mg} / \mathrm{ml}$ doxycycline dose was maintained for the rest of the studies.

We then investigated other signs of reprogramming at the gene expression level. A significant upregulation of Ki67, a marker of cell cycle activity, was found after 18 days of doxycycline-induced OKSM reprogramming (Figure 2e), in agreement with the increased proliferation rate that is observed during cell reprogramming (Polo et al., 2012). There was also a significant increase of Cdh1 and Epcam mRNA levels - markers of the mesenchymal-to-epithelial transition which is also characteristic of pluripotent reprogramming - at the same time point (Figure 2f). Lastly, the levels of ten-eleven translocation methylcytosine dioxygenases (TET) enzymes, known to catalyze DNA demethylation during cell reprogramming, were also significantly upregulated in reprogrammable mice during the course of the study (Figure S3). Lu et al have recently shown that active demethylation catalyzed by these enzymes is indeed necessary for OKS expression to translate into regenerative effects (Lu et al., 2020).

The architecture and morphology of reprogrammed cardiac tissues was also analyzed by transmission electron microscopy (TEM) for signs of reprogramming. The ventricular myocardium of reprogrammable mice that received normal drinking water (without doxycycline) showed the typical structure of aligned cardiac muscle fibers with organized sarcomeres and clearly distinguishable $A$ and I bands and $Z$ lines (Figure 2g, Figure S4b). However, ventricular tissues from reprogrammable mice that were offered doxycycline water for 18 days contained large areas filled with de-differentiated cardiomyocytes, as evidenced by the loss of fiber organization and sarcomere assembly (Figure $\mathbf{2 h}$ ). Sarcomere disassembly was also observed, albeit with less frequency, after 12 days of doxycycline administration (Figure S4c). On days 3 and 7 after the start of OKSM induction, no differences were observed, as compared to mice that did not received doxycycline (Figure S4c). 
a

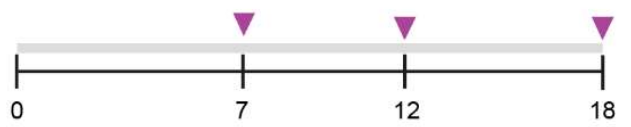

dox, $1 \mathrm{mg} / \mathrm{ml}$

$\nabla$ sacrifice

C

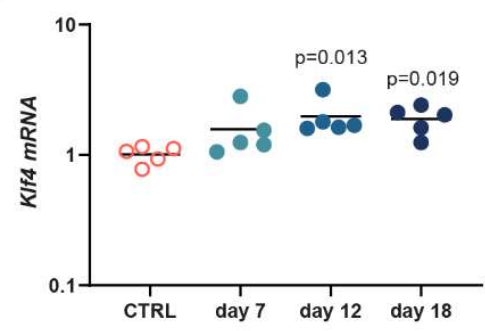

d

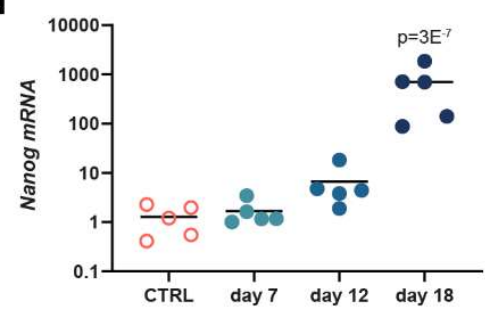

e

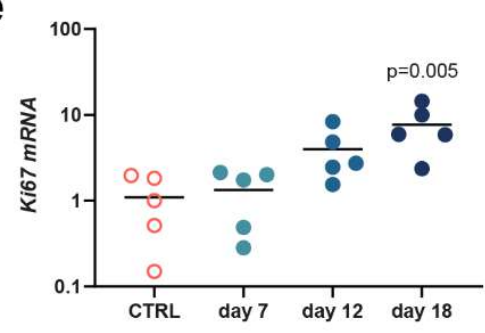

g

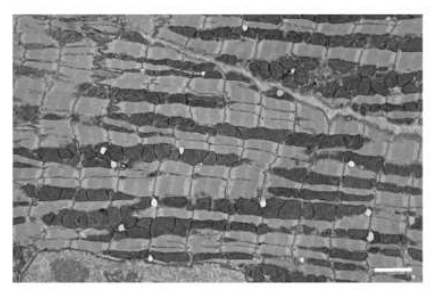

f

h b

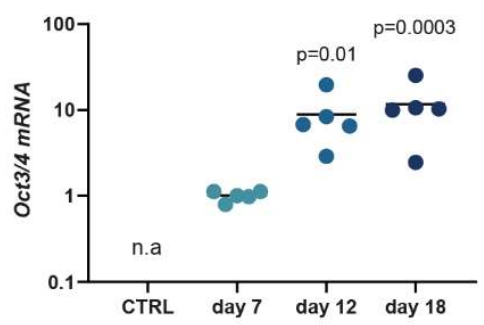

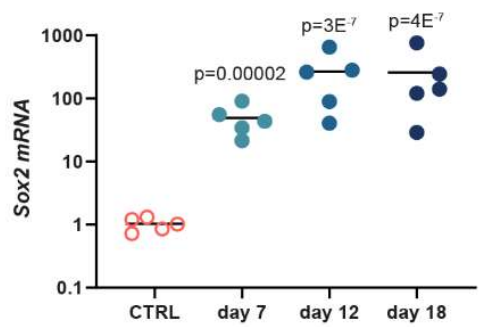
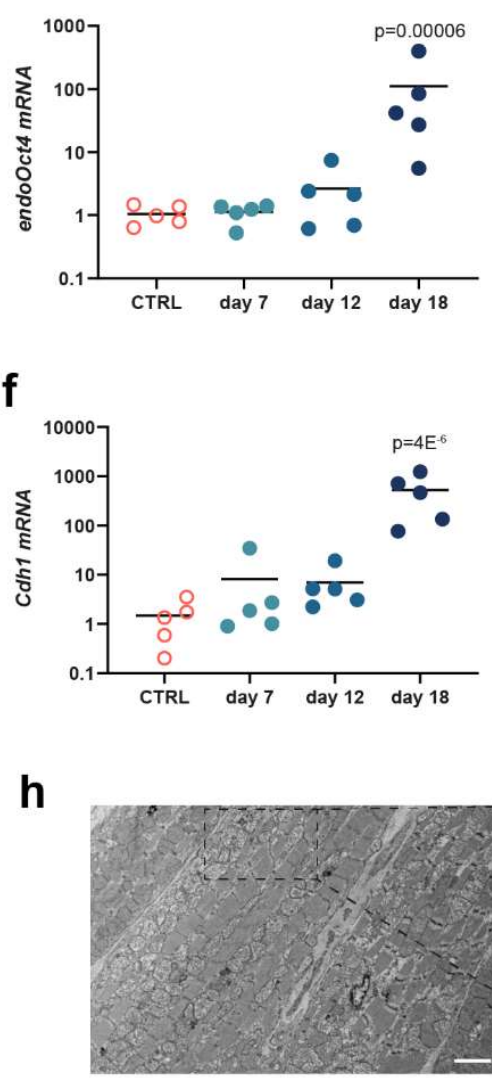
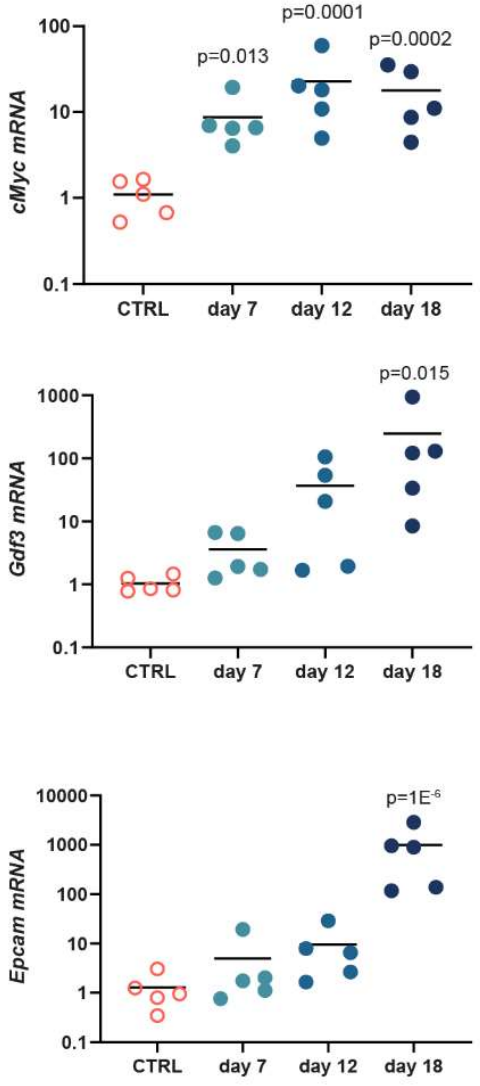

CTRL day 7 day 12 day 18

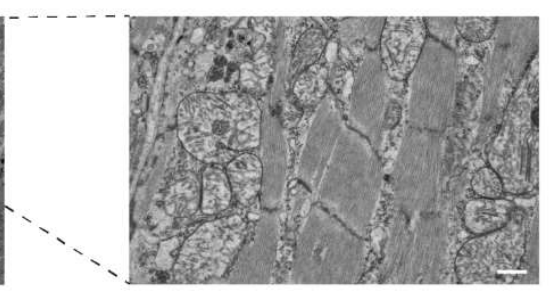

Figure 2. Eighteen days of dox-induced OKSM establish a gene expression program characteristic of pluripotent reprogramming in the myocardium and induces cardiomyocyte de-differentiation. (a) Schematic representation of experimental design. (b) mRNA levels of Oct $3 / 4$ reprogramming factor. (c) mRNA levels of other reprogrammable factors, KIf4, Sox2 and CMyc. (d) Gene expression levels of endogenous pluripotency markers. (e) Gene expression levels of Ki67. (f) mRNA levels of MET transition markers. Oct3/4 levels were 
normalized to those in Myh6-Cre ${ }^{+} \mathrm{Col1a}{ }^{\mathrm{OKSM}}$ mice (7 days of dox). The expression of all other genes investigated was normalized to that of control Myh6-Cre ${ }^{+} \mathrm{Col} 1 \mathrm{a} 1^{\mathrm{WT}}$ mice. Individual data points represent the fold change $\left(2^{\wedge-\Delta \Delta C t}\right)$ of each replicate $(n=5$ mice/group). Statistical analysis was performed by Welch ANOVA and Games-Howell post-hoc test (Gdf3 data) or one-way ANOVA and Tukey's post-hoc test (all other genes). $P$ values refer to statistical significance in relevance to the control group. (g) Representative TEM micrograph of cardiac ventricle tissue from reprogrammable mice that received normal drinking water, scale bar denotes $2 \mu \mathrm{m}$. (h) Representative TEM micrographs of cardiac ventricle tissue from reprogrammable mice administered with $1 \mathrm{mg} / \mathrm{ml}$ dox in the drinking water for 18 days. Scale bars represent $2 \mu \mathrm{m}$ (left) and $500 \mathrm{~nm}$ (closeup, right). The figure shows representative images from 5 random fields of view (FOV) and $n=2$ mice/group.

\section{Eighteen days of doxycycline-induced OKSM expression induces cardiomyocyte}

reprogramming to pluripotency. Based on the molecular and histological evidence of cardiomyocyte reprogramming found within the ventricular myocardium, we set out to confirm the cardiomyocyte origin and differentiation potential of in vivo reprogrammed cells via a teratoma assay, a gold-standard method to confirm functional pluripotency (Wesselschmidt, 2011). Reprogrammable (Myh6-Cre ${ }^{+} \mathrm{Col1a} 1^{\mathrm{OKSM}}, \mathrm{n}=7$ ) or control $\left(\mathrm{Myh6}_{-}-\mathrm{Cre}^{+} \mathrm{Col} 1 \mathrm{a} 1^{\mathrm{WT}}, \mathrm{n}=4\right)$ mice were administered doxycycline-supplemented water for 18 days, after which they were sacrificed and their hearts digested into single cell suspensions following a previously described Langendorfffree method (Ackers-Johnson et al., 2016) (Figure 3a). The cell suspensions obtained from atrial and ventricular tissues were processed separately and injected subcutaneously (s.c.) in opposite flanks of NU/J immunodeficient mice, to investigate the presence of pluripotent cells in all heart chambers. After 4 weeks, all cell suspensions obtained from atrial reprogrammed tissue (7/7) and $29 \%$ of those from the ventricles $(2 / 7)$ had generated tumor masses (Figure $\mathbf{3 b}$-d). Tumor masses were confirmed as teratomas with the presence of tissue types representative from all three germ layers: endoderm, mesoderm and ectoderm (Figures $\mathbf{3 e - g}$ ). No teratomas resulted from the inoculation of cardiac cell suspensions obtained from control, non-reprogrammable mice

(Figure 3b). These results confirmed that reprogrammed cells after 18 days of doxycyclineinduced OKSM expression are pluripotent at the functional level. The cardiomyocyte origin of such pluripotent cells was demonstrated by GFP positive staining within the teratomas (Figures 3f-g). 
a

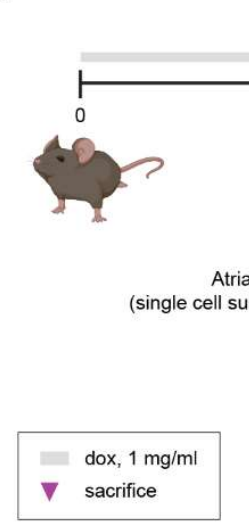

C

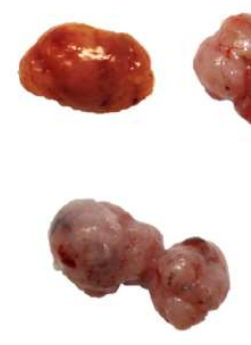

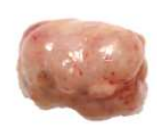

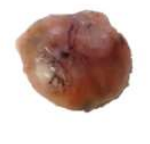

d

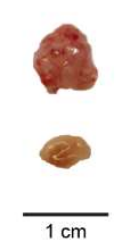

b

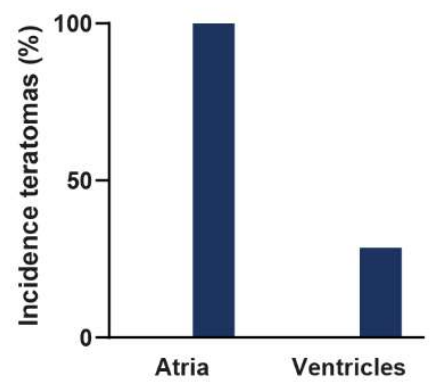

e

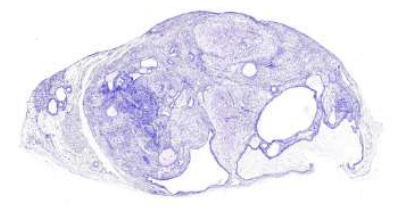

$\mathbf{f}$

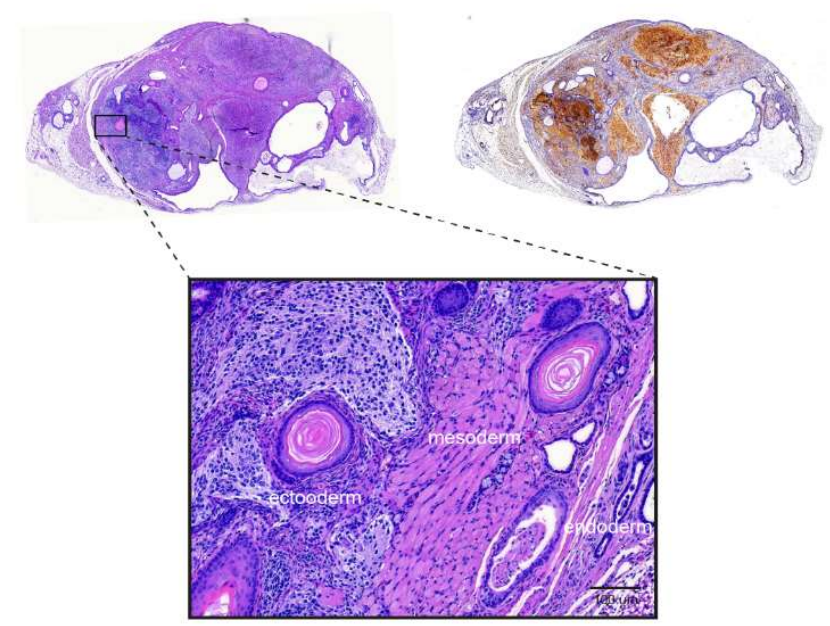

g

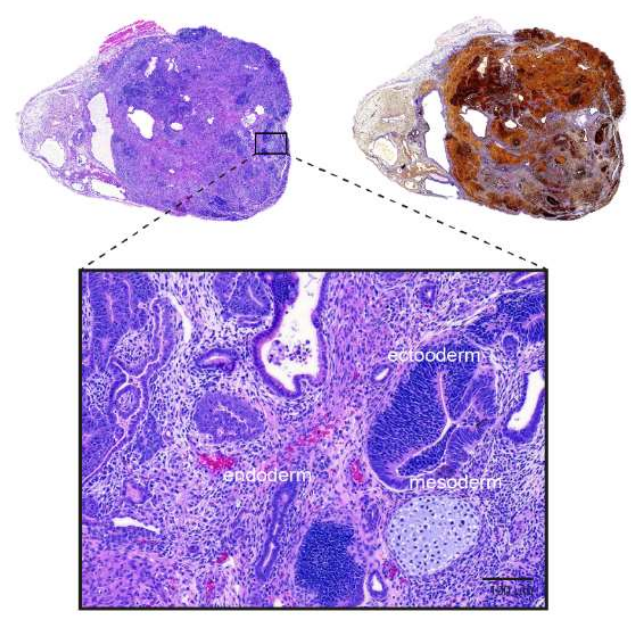

Figure 3. Eighteen days of dox-induced OKSM results in cardiomyocyte reprogramming to bona fide pluripotency. (a) Schematic representation depicting the workflow of the teratoma assay. Partially created with BioRender.com (b) Number of teratomas generated from atrial and ventricular single cell suspensions from reprogrammable (Myh6-Cre ${ }^{+} \mathrm{Col} 1 \mathrm{a} 1{ }^{\mathrm{OKSM}}$ ) and nonreprogrammable (Myh6-Cre ${ }^{+} \mathrm{Col1a1}{ }^{\mathrm{OKSM}}$ ) mice administered with dox for 18 days. Images of atrial (c) and ventricular (d) teratomas 4 weeks after s.c. injection of reprogrammed cardiac cells. Scale 
bars represent $1 \mathrm{~cm}$. (e) Negative control of GFP staining (no primary antibody) in a teratoma section (atrial origin). (f) Representative H\&E and GFP staining of a teratoma of atrial origin confirms trilineage contribution (high magnification insert, scale bar represents $100 \mu \mathrm{m}$ ) and cardiomyocyte origin (GFP positive staining). (g) Representative H\&E and GFP staining of a teratoma of ventricular origin confirms trilineage contribution (high magnification insert, scale bar represents $100 \mu \mathrm{m}$ ) and cardiomyocyte origin (GFP positive staining). Figure shows representative images, but trilineage contribution and GFP positive staining were confirmed in all teratomas.

Given the higher incidence of teratoma formation from reprogrammed cells in the atria (Figure 3b), changes in gene expression in this tissue were next analyzed upon the administration of doxycycline (Figure S5a). Analysis of Oct3/4 mRNA on day 12 after the start of doxycycline treatment demonstrated that this reprogramming factor is induced to similar levels in the ventricles and atria (Figure S5b) and the levels of endogenous pluripotency genes followed a very similar trend as seen in the ventricles (Figure S5c). Sarcomere disassembly was also obvious starting from day 12 after the initiation of the doxycycline treatment but, overall, there were more areas showing clear cardiac muscle fiber de-differentiation in the atria than in the ventricles (Figure S6). These results confirm that cardiomyocyte reprogramming takes place in both ventricular and atrial myocardium in this model, with likely a higher efficiency of reprogramming in the atria. The presence of reprogrammed cardiomyocytes in the atria is not surprising, as Myh6 is expressed in both atrial and ventricular tissue in mice, albeit with varying levels throughout development (Morkin, 2000).

\section{In vivo reprogrammed cardiomyocytes remain committed to pluripotency after 18 days of}

doxycycline-induced OKSM expression. We next set to investigate if reprogrammed cardiomyocytes (after 18 days of doxycycline-induced OKSM expression) remain committed to pluripotency after withdrawal of the drug and while maintained in their native microenvironment. Previous studies in mouse liver have shown that in vivo reprogrammed cells may exhibit molecular hallmarks of pluripotency and contribute to teratoma formation when transplanted into immune compromised hots but not when they remain in their native microenvironment (de Lazaro 
et al., 2014; Yilmazer et al., 2013). We administered doxycycline-supplemented water to reprogrammable (Myh6-Cre $\left.{ }^{+} \mathrm{Col1a}{ }^{\mathrm{OKSM}}\right)$ and control (Myh6-Cre-Col1a1 ${ }^{\mathrm{OKSM}}$, Myh6$\mathrm{Cre}^{+} \mathrm{Col} 1 \mathrm{a} 1^{\mathrm{WT}}$ ) mice for 18 days, followed by withdrawal of the drug (Figure 4a). No significant changes in body weight were observed for the first 30 days of the study (Figure 4b), after which reprogrammable mice had to be euthanized for humane reasons (mainly showing difficulty in breathing and lethargy). The entire group succumbed within 58 days of the start of doxycycline treatment (Figure 4d). Upon necropsy investigation, 75\% (6/8) of Myh6-Cre ${ }^{+} \mathrm{Col} 1 \mathrm{a} 1^{\mathrm{OKSM}}$ mice showed clear tumor masses growing out of the heart (Figure 4c, e). One mouse in that group was found dead on day 18 and another one had to be euthanized on day 58 due to a spontaneous tumor growing on the head, but histological investigation did not reveal any signs of dysplasia or teratoma formation in the heart in either of those cases (data not shown). On the contrary, most mice from control (non-reprogrammable) groups survived for the 240-day duration of the study (Figure 4d) and the one mouse per group that was found dead did not show any evidence of tumorigenesis of dysplasia (Figure $\mathbf{4 c}$ and data not shown). Histological investigation of reprogrammed hearts confirmed trilineage contribution (i.e. teratoma identity) in tumor masses sprouting from the heart (Figure 4e, ii-iii) and other areas of dysplasia were found in atrial and ventricular tissues (Figure $\mathbf{4 e}, \mathbf{i v}-\mathbf{v}$ ). We then studied the gene and protein expression patterns in the teratomas in order to lineage trace their origin. Myh6 (Figure $\mathbf{4 f}$ ) and $c T n T$ (Figure $\mathbf{4 g}$ ) mRNA levels were significantly downregulated within large teratomas sprouting from the heart, compared to their respective ventricular tissue. However, GFP expression levels were maintained (Figure 4h). These results suggest that cells within teratomas originated from cardiomyocytes, and thus expressed GFP, but lost cardiomyocyte identity (Myh6 and cTnt expression) upon reprogramming. These observations were confirmed by immunohistochemistry (Figure 4i-j). Teratomas stained strongly for GFP and showed clusters of proliferative $\mathrm{Ki} 67^{+}$cells, while the expression of cTNT was extremely low and not uniformly detected. In addition, the expression of OKSM factors (Figure S7a), endogenous pluripotency markers (Figure S7b) and a significant 
bioRxiv preprint doi: https://doi.org/10.1101/2021.12.22.473302; this version posted December 23, 2021. The copyright holder for this preprint

(which was not certified by peer review) is the author/funder, who has granted bioRxiv a license to display the preprint in perpetuity. It is made available under aCC-BY-NC-ND 4.0 International license.

upregulation of Ki67 (Figure S7c) was confirmed in teratomas and ventricular myocardium of reprogrammed hearts. Overall, these results confirm that after 18 days of doxycycline-induced reprogramming, mouse cardiomyocytes are committed to pluripotency, independently of induced OKSM expression and even when maintained in the native microenvironment of the adult mouse myocardium. 
a
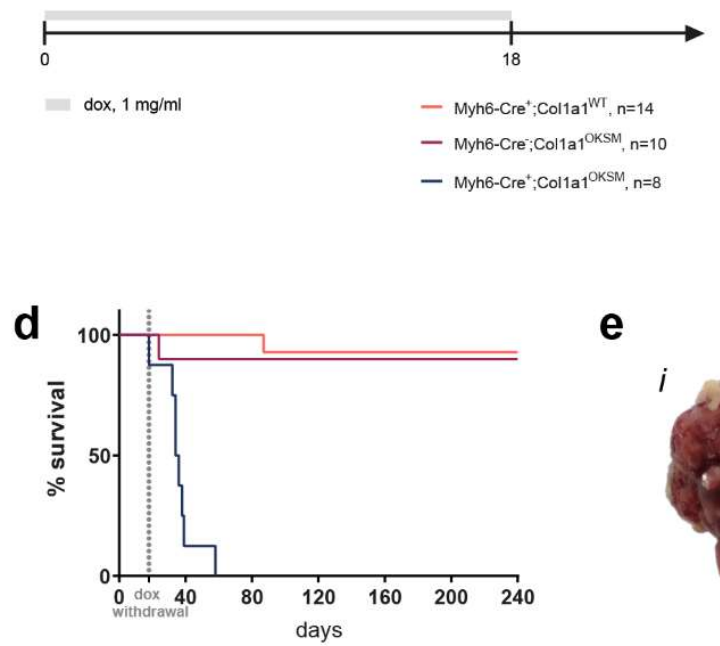

f

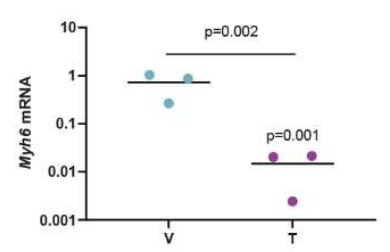

h

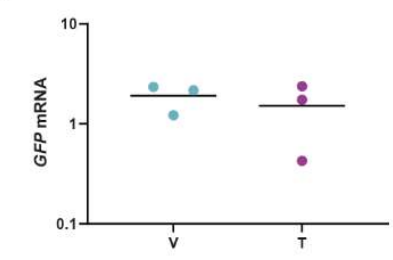

j

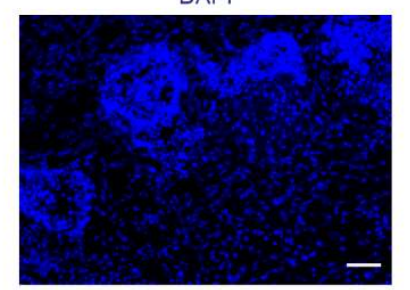

e

g

i b

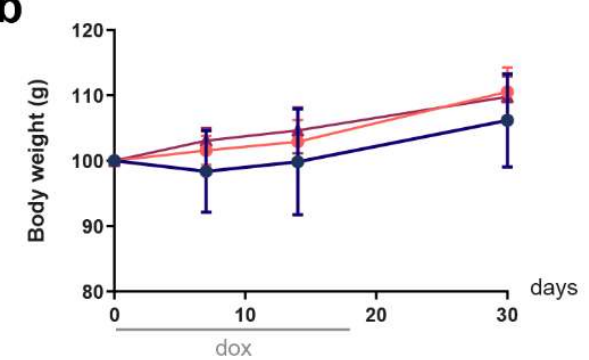

C

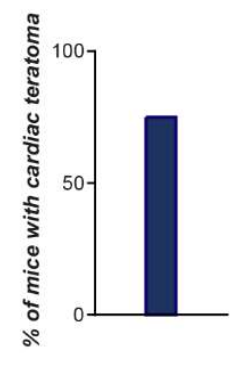

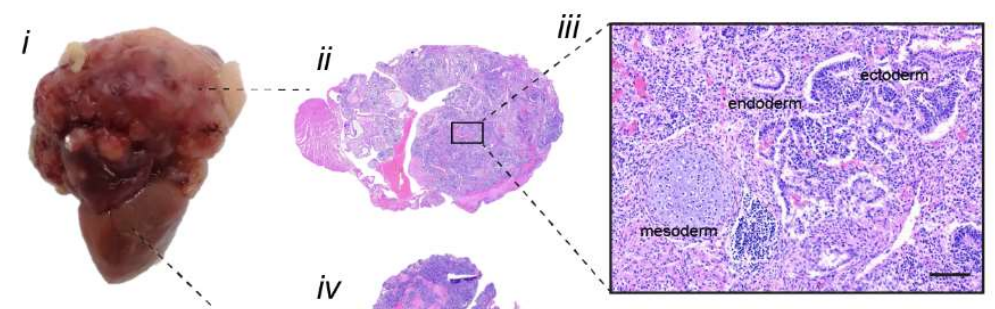
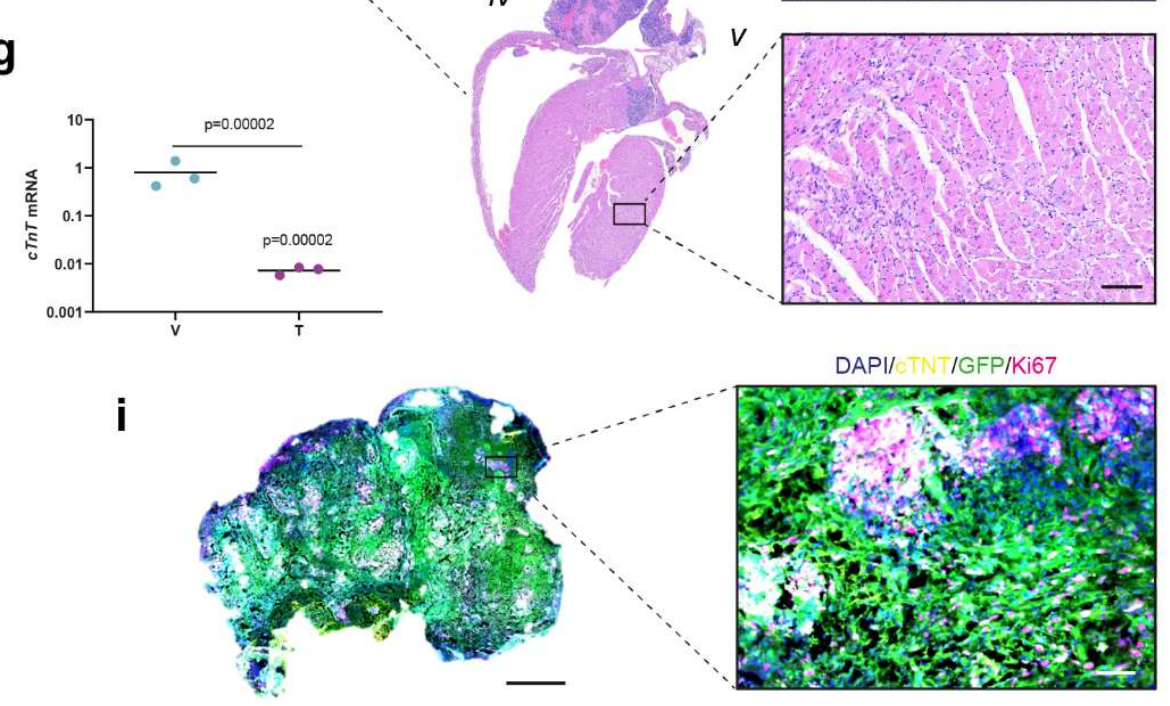

Figure 4. Doxycycline-induced expression of OKSM for 18 days generates teratomas in the heart of reprogrammable mice. (a) Schematic representation of experimental design. (b) Changes in body weight upon administration of $1 \mathrm{mg} / \mathrm{ml}$ dox in the drinking water of reprogrammable (Myh6 $\left.{ }^{+} \mathrm{Col} 1 \mathrm{a}^{\mathrm{OKSM}}\right)$ and control $\left(\mathrm{Myh}^{+} \mathrm{Col} 1 \mathrm{a} 1^{\mathrm{WT}}\right.$; Myh6-Col1a1 $\left.{ }^{\mathrm{OKSM}}\right)$ mice. (c) Incidence of teratoma formation upon doxycycline treatment. Teratomas were only found in reprogrammable mice. (d) Survival of reprogrammable and control mice after 18 days on doxycycline treatment. (e) Representative teratoma in the heart of a reprogrammable mouse (i): whole tissue section (ii) and close-up at 10X magnification (iii) of teratoma H\&E staining; whole 
tissue section (iv) and close-up at 10X magnification (v) of the four chambers of the heart. Scale bars represent $100 \mu \mathrm{m}$. Gene expression levels of Myh6 (f), cTnT (g), and GFP (h) in cardiac teratomas and ventricular myocardium. Gene expression data are normalized to that of Myh6 ${ }^{+} \mathrm{Col} 1 \mathrm{a} 1^{\mathrm{WT}}$ cardiac tissue exposed to the same doxycycline regime. Individual data points represent the fold change $\left(2^{\wedge-\Delta \Delta C t}\right)$ of each replicate $(n=5$ mice/group). Statistical analysis was performed by one-way ANOVA and Tukey's post-hoc test. $P$ values refer to statistical significance in relevance to the control group or between ventricle and teratoma (Myh6 and cTnT, denoted with a line between the two groups). (i) Triple immunofluorescence for cTNT, GFP and Ki67 in cardiac teratomas. Scale bar represents $1 \mathrm{~mm}$ (whole section) and $50 \mu \mathrm{m}$ (close-up at 10X magnification). (j) Separate channels for triple immunofluorescence. Scale bars represent $50 \mu \mathrm{m}$.

\section{DISCUSSION}

We demonstrate here that Cre recombination under the control of a cell type-specific promoter and conditional, doxycycline-inducible regulation of gene expression can be combined to achieve cardiomyocyte-specific expression of OKSM and reprogramming. In addition, our work confirms that adult mouse cardiomyocytes can be fully reprogrammed to the pluripotent state. This possibility had remained elusive in earlier studies, likely due to a combination of low OKSM expression, poor temporal control over the latter, and use of viral vectors with promiscuous tropism to induce OKSM (Kisby et al., 2021a). The ability to reverse the fully differentiated state of adult mammalian cardiomyocytes to bona fide pluripotency is an important observation, since there is typically an inverse relationship between the differentiation status of a cell and its capacity to undergo reprogramming (Eminli et al., 2009; Polo et al., 2010; Tan et al., 2011) and reports of post-mitotic cells undergoing reprogramming are scarce, mainly limited to transdifferentiation events (De la Rossa et al., 2013; Rouaux and Arlotta, 2013) or require p53 suppression to force cell proliferation (Kim et al., 2011).

Our findings and those in a recent publication (Chen et al., 2021) suggest a new avenue for cardiac regeneration through cardiomyocyte reprogramming to a proliferative state. Chen and colleagues also demonstrated adult cardiomyocyte reprogramming to pluripotency, using a similar cardiomyocyte-specific reprogrammable mouse model (double mutant, and under the control of a different cardiomyocyte-specific promoter, by crossing the Myl2-Cre mouse with a 
conditional and inducible OKSM strain) (Chen et al., 2021). Their work also demonstrated that cardiomyocytes can be partially reprogrammed to a proliferative but not pluripotent state with a neonatal-like gene expression profile by limiting the duration of OKSM expression to 6 days, and that partial cardiomyocyte reprogramming is associated with an improvement of cardiac regeneration after myocardial infarction (Chen et al., 2021). This approach adds to other gene therapies also proposed to stimulate endogenous cardiomyocyte proliferation, including those leveraging cell cycle regulators such as Cyclin D2 (Shapiro et al., 2014), metabolic regulators such as ERRB2 (D'Uva et al., 2015) or non-coding RNAs that regulate cardiomyocyte regeneration in other species (Aguirre et al., 2014). An advantage of OKSM-induced reprogramming is that, being a stepwise process, its potential may not be limited to the repopulation of lost cardiomyocytes. Shorter OKSM expression or the absence of cMyc have shown to limit reprogramming in vivo to the resetting of ageing signatures without proliferation or loss of cell identity, and this rejuvenation has shown to increase overall fitness and improve the outcomes of subsequent tissue damage (Lu et al., 2020; Ocampo et al., 2016). Thus, OKSM partial cardiomyocyte reprogramming may also offer opportunities to treat and prevent ailments of the aging heart that do not necessarily present with extensive cardiomyocyte death.

Cell type-specific reprogrammable mouse models may enable higher mechanistic depth of studies, for example, to elucidate key cell types required to be reprogrammed for the successful regeneration of a specific tissue, or to investigate the crosstalk between reprogrammed and nonreprogrammed cells, but examples of these models are still scarce in the scientific literature. Besides the work of Chen et al and this study, only Wang and colleagues have recently reported on skeletal myofiber and satellite cell-specific reprogrammable mouse models (Wang et al., 2021). Their work uncovered a paracrine effect of partially reprogrammed myofibers on the skeletal muscle stem cell niche as the direct mediator of regeneration, which could not have been elucidated using traditional reprogrammable mice with ubiquitous OKSM expression. Future research in this field will also likely focus on the development of clinically translatable alternative 
approaches. Doxycycline-inducible adeno-associated viral vectors have shown promise towards this goal thanks to tissue-specific tropism and control over the duration of gene expression (Lu et al., 2020) but they still do not offer tropism with cell type resolution unless combined with Cre transgenic lines. In parallel, extensive research on small molecule drugs to replace OKSM is underway (Kim et al., 2020).

In sum, our findings support the ability of adult cardiomyocytes to be reprogrammed to pluripotency, and more broadly the potential of in vivo cell reprogramming for regenerative medicine and a platform with high control over OKSM expression to enable further mechanistic studies.

\section{EXPERIMENTAL PROCEDURES}

Mice. All animal procedures were performed in accordance with the Harvard University Faculty of Arts and Sciences (FAS) Institutional Animal Care and Use Committee (IACUC) guidelines. B6.FVB-Tg(Myh6-cre)2182Mds/J mice express constitutively active Cre recombinase enzyme under the control of the Myh6 promoter through a transgene insertion (Agah et al., 1997), and were obtained from the Jackson Laboratory (Cat \#011038). B6.CgGt(ROSA)26Sor ${ }^{t m 1(r T A, E G F P) N a g y / J}$ mice contain a floxed (flanking loxP) Pgk-neo-pA cassette and a rtTA-IRES-EGFP-pA cassette inserted into intron 1 of the Rosa26 locus (Belteki et al., 2005), and were purchased from the Jackson Laboratory (Cat \#005670). B6;129S4-Col1a1 ${ }^{\text {tm1 1(teto-Pou5f1,-Klf4,- }}$ Sox2,-Myc)Hoch/J mice have a targeted mutation in the Col1a1 gene. The OKSM cassette consisting of four mouse reprogramming genes, Oct3/4, KIf4, Sox2, and $c M y c$, is expressed under the control of the bi-directional tet-responsive element (tetO) with CMV minimal enhancer-less promoter (Stadtfeld et al., 2010). These mice were a kind gift from Prof Konrad Hochendlinger (Massachusetts General Hospital and Harvard University). The three strains above were bred in house to generate cardiomyocyte-specific, inducible reprogrammable mice. The rtTA-IRESEGFP-pA was kept in homozygosis while the Cre recombinase and OKSM modifications were maintained as heterozygotes following advice from donating investigators for optimal breeding. For simplicity, triple transgenic reprogrammable mice are named Myh6-Cre ${ }^{+} \mathrm{Col1a}{ }^{\mathrm{OKSM}}$ or cardiomyocyte-specific reprogrammable mice hereinafter. Myh6-Cre ${ }^{+} \mathrm{Col} 1 \mathrm{a} 1^{\mathrm{WT}}$, Myh6-CreCol1a1 ${ }^{\text {OKSM }}$ and Myh6-Cre-Col1a1 ${ }^{\mathrm{WT}}$ mice were included as controls throughout the study, as specified for each experiment. Mice of 8-12 weeks of age and of both sexes were used for the studies. 6-week-old Nu/J mice for the teratoma assay were obtained from The Jackson Laboratory (Cat \#00219).

Doxycycline administration. Doxycycline (Sigma) was added in the drinking water at $1 \mathrm{mg} / \mathrm{ml}$, unless otherwise specified. To improve taste, water was supplemented with $7.5 \%$ sucrose. Mice 
were let to drink water ab libitum, which was kept in amber water bottles to protect the drug from degradation. Fresh dox water was replaced weekly for the duration of the experiment.

Fluorescence activated cell sorting (FACS) of cardiac cells. Hearts from Myh6$\mathrm{Cre}^{+} \mathrm{Col1a}{ }^{\mathrm{OKSM}}(\mathrm{n}=5)$ or Myh6-Cre-Col1a1 ${ }^{\mathrm{WT}}$ mice $(n=2)$ were digested into single cell suspensions following a Langendorff-free method as previously described (Ackers-Johnson et al., 2016) and filtered through a $100 \mu \mathrm{m}$ strainer (Corning). Cardiac single cell suspensions were stained with cell-specific antibodies (Supplementary Table 1) at the concentrations recommended by the manufacturers and sorted on a MoFlo Astrios with a $200 \mu \mathrm{m}$ nozzle at 9psi (Beckman Coulter) at the Bauer Core Facility of Harvard University.

Sorting of cardiac cell types by centrifugation and magnetic separation. Myh6$\mathrm{Cre}^{+} \mathrm{Col} 1 \mathrm{a}{ }^{\mathrm{OKSM}}$ mice $(\mathrm{n}=5)$ were administered $1 \mathrm{mg} / \mathrm{ml}$ doxycycline in the drinking water for 7 days, followed by euthanasia and digestion of the heart as previously described (Ackers-Johnson et al., 2016). Cardiac myocytes were isolated by two rounds of centrifugation (5 min each) at 30 $\mathrm{g}$. The non-myocyte fraction was then subjected to another round of centrifugation at $30 \mathrm{~g}$ to remove any remaining cardiomyocytes. Cardiac endothelial cells were subsequently isolated from the rest of non-myocytes by magnetic cell sorting using a neonatal cardiac endothelial cell isolation kit (Miltenyi) and following the manufacturer's instructions. Isolated cell types were further lysed in $1 \mathrm{ml}$ Purezol (Biorad) to proceed with RNA extraction.

Gene expression by real-time RT-qPCR. Total RNA was extracted from sorted cardiac cells or from cardiac tissues (atria or ventricle) using the Aurum total RNA fatty and fibrous tissues kit (Biorad) and following the manufacturer's instructions. Tissue samples were homogenized in 1 $\mathrm{ml}$ Purezol (Biorad) on a gentleMACS® Octo dissociator (Miltenyi) using M tubes (Miltenyi). RNA quantity and quality were determined by spectrophotometry (Nanodrop). $0.1 \mu \mathrm{g}$ RNA (sorted cells) or $1 \mu \mathrm{g}$ RNA (tissues) was used to prepare cDNA with the iScript ${ }^{\mathrm{TM}} \mathrm{CDNA}$ synthesis kit (Biorad) with the following protocol: $5^{\circ} \mathrm{C}$ for 5 minutes (min), $46^{\circ} \mathrm{C}$ for $20 \mathrm{~min}, 95^{\circ} \mathrm{C}$ for $1 \mathrm{~min}$. iTaq Universal SYBR green (Biorad) was used to perform real-time qPCR reaction on a CFX96 real-time PCR system (Biorad), following the manufacturer's protocol: $95^{\circ} \mathrm{C}$ for 30 seconds (s), followed by 40 cycles of $95^{\circ} \mathrm{C}$ for $5 \mathrm{~s}, 60^{\circ} \mathrm{C}$ for $30 \mathrm{~s}$. A melt curve from $65^{\circ} \mathrm{C}$ to $95^{\circ} \mathrm{C}$ was performed at the end of the run to ensure amplification of a single product. Mapk1 and Rps13, identified as the most stable reference genes during cardiac reprogramming in a previous report(Kisby et al., 2021a), were used for data normalization. The list of primers used in this study is found in Supplementary Table 2. Real-time RT-qPCR studies were performed in compliance with the Minimum Information for Publication of Quantitative Real-Time PCR Experiments (MIQE) Guidelines(Bustin et al., 2009). For statistical analysis, $\Delta \Delta C T$ values were used.

Transmission electron microscopy (TEM). Myh6-Cre ${ }^{+} \mathrm{Col1a} 1^{\mathrm{OKSM}}$ mice were given drinking water with $1 \mathrm{mg} / \mathrm{ml}$ doxycycline for 3, 7, 12 or 18 days, or otherwise provided with unadulterated drinking water ( $n=2$ mice/group). At the end of the doxycycline treatment, mice were euthanized and $0.5 \mathrm{ml}$ of $1 \mathrm{M} \mathrm{KCl}$ was injected through the left ventricle to arrest the heart in diastole, followed by perfusion with $1 \mathrm{ml}$ of TEM fixative $(2.5 \%$ glutaraldehyde and $2 \%$ formaldehyde in $0.1 \mathrm{M}$ Sodium cacodylate buffer, $\mathrm{pH} 7.4$ ). Hearts were then cut into 1-2 mm cubes and incubated in fresh fixative 
for at least 2 hours (h) at room temperature. Samples were processed for TEM imaging at the Electron Microscopy Facility (Harvard Medical School) following standard techniques. 5 random FOV at $3000 \mathrm{X}$ and 5 random FOV at $10000 \mathrm{X}$ were imaged from each heart sample $(\mathrm{n}=2$ mice per group).

Teratoma assay. Myh6-Cre ${ }^{+} \mathrm{Col1a}{ }^{\mathrm{OKSM}}(\mathrm{n}=7)$ and Myh6-Cre ${ }^{+} \mathrm{Col1a} 1^{\mathrm{WT}}(\mathrm{n}=4)$ mice were given drinking water with $1 \mathrm{mg} / \mathrm{ml}$ doxycycline for 18 days and euthanized at the end of the treatment. Hearts were digested into a single cell suspension as described above but, for each heart, cells from the ventricles and from the atria were processed separately. The cells were filtered through a $100 \mu \mathrm{m}$ strainer (Corning) and washed three times by centrifugation ( $450 \mathrm{~g}, 5 \mathrm{~min}$ ) in ice-cold PBS. Cell pellets were finally resuspended in a $100 \mu \mathrm{l}$ 1:1 mix of ice-cold PBS and reduced-growth factor Matrigel (Corning) and injected subcutaneously in 6-week-old Nu/J mice (\#00219, The Jackson Laboratory). The atrial and ventricular cell suspension of each digested heart were injected in the left and right dorsal flank of a Nu/J mouse, respectively. Teratomas were allowed to form for 4 weeks, and subsequently dissected and fixed in $10 \%$ formalin prior to histological analysis by hematoxylin and eosin (H\&E) staining and GFP immunohistochemistry following routine protocols, which was performed at iHisto (Boston, USA). Details of antibodies used in staining studies in this work are shown in Supplementary Table 3.

\section{Investigation of the fate of in vivo reprogrammed cardiomyocytes (generation of teratomas} in the heart). Myh6-Cre+Col1a1 ${ }^{\mathrm{OKSM}}(\mathrm{n}=8)$, Myh6-Cre+Col1a1 ${ }^{\mathrm{WT}}(\mathrm{n}=14)$ and Myh6-CreCol1a1 ${ }^{\text {OKSM }}(\mathrm{n}=10)$ mice were given drinking water with $1 \mathrm{mg} / \mathrm{ml}$ doxycycline for 18 days and then returned to unadulterated drinking water. Mice were closely monitored for changes in body weight and for signs of distress. When mice were found dead or with evident signs of distress, hearts were dissected and washed in PBS. Tissue samples were collected and processed for gene expression (stored in RNA later until RNA isolation was performed as described above) and for histological evaluation.

Cryosectioning and immunofluorescence. Cardiac teratomas were washed in PBS, embedded in OCT and flash-frozen in isopentane (Sigma) pre-cooled in liquid nitrogen. $10 \mu \mathrm{m}$ thick sections were obtained with a cryostat (Leica) and processed for immunofluorescence following a standard protocol. The antibodies and dilutions used in this study are listed in Supplementary Table 3. Images were acquired with an Axio Scan slide scanner (Zeiss) and processed with QuPath open software (version 0.2.3) (Bankhead et al., 2017).

Statistical analysis. Statistical analysis was performed with IBM SPSS $®$ Statistics software (version 25). The specific tests applied for each study are described in the respective figure legend. In brief, when dealing with multiple comparisons, Levene's test of homogeneity of variances was first applied. one-way ANOVA and Tukey's post-hoc test were used when the variances were homogeneous while Welch ANOVA and Games-Howell post-hoc test were used when the variances of the samples were significantly different. 


\section{ACKNOWLEDGEMENTS}

We thank Prof Konrad Hochedlinger (Massachusetts General Hospital and Harvard University) for kindly providing B6;129S4-Col1a1tm1(tetO-Pou5f1,-Kff4,-Sox2,-Myc)Hoch/J mice. Electron microscopy imaging, consultation and processing of samples were performed at the HMS Electron Microscopy Facility. We thank Zachary Niziolek and the Bauer Core Facility at Harvard University for consultation, performance and analysis of FACS experiments, and staff at the Harvard Bioimaging Facility for assistance with imaging experiments. IdL wishes to thank Matthew Pezone and Dr Maxence Dellacherie for occasional help maintaining the reprogrammable mice colony.

\section{AUTHOR CONTRIBUTIONS}

IdL and DM conceived the study. IdL performed all the experiments. TOS contributed to some RT-qPCR experiments and CMT assisted with sample processing for the teratoma study. IdL and DM discussed the data and wrote the manuscript. All co-authors provided their edits to the manuscript.

\section{CONFLICT OF INTERESTS}

The authors declare no conflict of interests. 


\section{References}

1. Abad, M., Mosteiro, L., Pantoja, C., Canamero, M., Rayon, T., Ors, I., Grana, O., Megias, D., Dominguez, O., Martinez, D., et al. (2013). Reprogramming in vivo produces teratomas and iPS cells with totipotency features. Nature 502, 340-345.10.1038/nature12586.

2. Ackers-Johnson, M., Li, P.Y., Holmes, A.P., O'Brien, S.M., Pavlovic, D., and Foo, R.S. (2016). A Simplified, Langendorff-Free Method for Concomitant Isolation of Viable Cardiac Myocytes and Nonmyocytes From the Adult Mouse Heart. Circulation research 119, 909-920.

10.1161/circresaha.116.309202.

3. Agah, R., Frenkel, P.A., French, B.A., Michael, L.H., Overbeek, P.A., and Schneider, M.D. (1997). Gene recombination in postmitotic cells. Targeted expression of Cre recombinase provokes cardiacrestricted, site-specific rearrangement in adult ventricular muscle in vivo. J Clin Invest 100, 169-179. 10.1172/JCl119509.

4. Aguirre, A., Montserrat, N., Zacchigna, S., Nivet, E., Hishida, T., Krause, M.N., Kurian, L., Ocampo, A., Vazquez-Ferrer, E., Rodriguez-Esteban, C., et al. (2014). In vivo activation of a conserved microRNA program induces mammalian heart regeneration. Cell Stem Cell 15, 589-604.

10.1016/j.stem.2014.10.003.

5. Alle, Q., Le Borgne, E., Bensadoun, P., Lemey, C., Béchir, N., Gabanou, M., Estermann, F., BertrandGaday, C., Pessemesse, L., Toupet, K., et al. (2021). A single short reprogramming early in life improves fitness and increases lifespan in old age. bioRxiv, 2021.2005.2013.443979. 10.1101/2021.05.13.443979.

6. Bankhead, P., Loughrey, M.B., Fernández, J.A., Dombrowski, Y., McArt, D.G., Dunne, P.D., McQuaid, S., Gray, R.T., Murray, L.J., Coleman, H.G., et al. (2017). QuPath: Open source software for digital pathology image analysis. Scientific Reports 7, 16878. 10.1038/s41598-017-17204-5.

7. Belteki, G., Haigh, J., Kabacs, N., Haigh, K., Sison, K., Costantini, F., Whitsett, J., Quaggin, S.E., and Nagy, A. (2005). Conditional and inducible transgene expression in mice through the combinatorial use of Cre-mediated recombination and tetracycline induction. Nucleic Acids Res 33, e51. 10.1093/nar/gni051.

8. Bustin, S.A., Benes, V., Garson, J.A., Hellemans, J., Huggett, J., Kubista, M., Mueller, R., Nolan, T., Pfaffl, M.W., Shipley, G.L., et al. (2009). The MIQE guidelines: minimum information for publication of quantitative real-time PCR experiments. Clin Chem 55, 611-622. 10.1373/clinchem.2008.112797.

9. Chen, Y., Lüttmann Felipe, F., Schoger, E., Schöler Hans, R., Zelarayán Laura, C., Kim, K.-P., Haigh Jody, J., Kim, J., and Braun, T. (2021). Reversible reprogramming of cardiomyocytes to a fetal state drives heart regeneration in mice. Science 373, 1537-1540. 10.1126/science.abg5159.

10. D'Uva, G., Aharonov, A., Lauriola, M., Kain, D., Yahalom-Ronen, Y., Carvalho, S., Weisinger, K., Bassat, E., Rajchman, D., Yifa, O., et al. (2015). ERBB2 triggers mammalian heart regeneration by promoting cardiomyocyte dedifferentiation and proliferation. Nature Cell Biology 17, 627-638. 10.1038/ncb3149.

11. David, L., and Polo, J.M. (2014). Phases of reprogramming. Stem Cell Research 12, 754-761. https://doi.org/10.1016/j.scr.2014.03.007.

12. De la Rossa, A., Bellone, C., Golding, B., Vitali, I., Moss, J., Toni, N., Luscher, C., and Jabaudon, D. (2013). In vivo reprogramming of circuit connectivity in postmitotic neocortical neurons. Nat Neurosci 16, 193-200. 10.1038/nn.3299.

13. de Lazaro, I., Bussy, C., Yilmazer, A., Jackson, M.S., Humphreys, N.E., and Kostarelos, K. (2014). Generation of induced pluripotent stem cells from virus-free in vivo reprogramming of BALB/c mouse liver cells. Biomaterials 35, 8312-8320. 10.1016/j.biomaterials.2014.05.086. 
14. de Lazaro, I., Cossu, G., and Kostarelos, K. (2017). Transient transcription factor (OSKM) expression is key towards clinical translation of in vivo cell reprogramming. EMBO Mol Med.

10.15252/emmm.201707650.

15. de Lazaro, I., and Kostarelos, K. (2014). In vivo cell reprogramming to pluripotency: exploring a novel tool for cell replenishment and tissue regeneration. Biochemical Society Transactions 42, 711-716. https://doi.org/10.1042/BST20140012

16. de Lazaro, I., Yilmazer, A., Nam, Y., Qubisi, S., Razak, F.M.A., Degens, H., Cossu, G., and Kostarelos, K. (2019). Non-viral, Tumor-free Induction of Transient Cell Reprogramming in Mouse Skeletal Muscle to Enhance Tissue Regeneration. Mol Ther 27, 59-75. 10.1016/j.ymthe.2018.10.014.

17. Doeser, M.C., Scholer, H.R., and Wu, G. (2018). Reduction of Fibrosis and Scar Formation by Partial Reprogramming In Vivo. Stem Cells. 10.1002/stem.2842.

18. Eminli, S., Foudi, A., Stadtfeld, M., Maherali, N., Ahfeldt, T., Mostoslavsky, G., Hock, H., and Hochedlinger, K. (2009). Differentiation stage determines potential of hematopoietic cells for reprogramming into induced pluripotent stem cells. Nature Genetics 41, 968-976. 10.1038/ng.428.

19. Eschenhagen, T., Bolli, R., Braun, T., Field, L.J., Fleischmann, B.K., Frisén, J., Giacca, M., Hare, J.M., Houser, S., Lee, R.T., et al. (2017). Cardiomyocyte Regeneration: A Consensus Statement. Circulation 136, 680-686. 10.1161/CIRCULATIONAHA.117.029343.

20. Kim, J., Lengner, C.J., Kirak, O., Hanna, J., Cassady, J.P., Lodato, M.A., Wu, S., Faddah, D.A., Steine, E.J., Gao, Q., et al. (2011). Reprogramming of postnatal neurons into induced pluripotent stem cells by defined factors. Stem cells (Dayton, Ohio) 29, 992-1000. 10.1002/stem.641.

21. Kim, Y., Jeong, J., and Choi, D. (2020). Small-molecule-mediated reprogramming: a silver lining for regenerative medicine. Experimental \& Molecular Medicine 52, 213-226. 10.1038/s12276-020-0383-3.

22. Kisby, T., de Lázaro, I., Fisch, S., Cartwright, E.J., Cossu, G., and Kostarelos, K. (2021a). Adenoviral Mediated Delivery of OSKM Factors Induces Partial Reprogramming of Mouse Cardiac Cells In Vivo. Advanced Therapeutics 4, 2000141. https://doi.org/10.1002/adtp.202000141.

23. Kisby, T., de Lázaro, I., Stylianou, M., Cossu, G., and Kostarelos, K. (2021b). Transient reprogramming of postnatal cardiomyocytes to a dedifferentiated state. PLOS ONE 16, e0251054. 10.1371/journal.pone.0251054.

24. Lu, Y., Brommer, B., Tian, X., Krishnan, A., Meer, M., Wang, C., Vera, D.L., Zeng, Q., Yu, D., Bonkowski, M.S., et al. (2020). Reprogramming to recover youthful epigenetic information and restore vision. Nature 588, 124-129. 10.1038/s41586-020-2975-4.

25. Morkin, E. (2000). Control of cardiac myosin heavy chain gene expression. Microscopy research and technique 50, 522-531. 10.1002/1097-0029(20000915)50:6<522::Aid-jemt9>3.0.Co;2-u.

26. Ocampo, A., Reddy, P., Martinez-Redondo, P., Platero-Luengo, A., Hatanaka, F., Hishida, T., Li, M., Lam, D., Kurita, M., Beyret, E., et al. (2016). In Vivo Amelioration of Age-Associated Hallmarks by Partial Reprogramming. Cell 167, 1719-1733 e1712. 10.1016/j.cell.2016.11.052.

27. Polo, Jose M., Anderssen, E., Walsh, Ryan M., Schwarz, Benjamin A., Nefzger, Christian M., Lim, Sue M., Borkent, M., Apostolou, E., Alaei, S., Cloutier, J., et al. (2012). A Molecular Roadmap of Reprogramming Somatic Cells into iPS Cells. Cell 151, 1617-1632. http://dx.doi.org/10.1016/j.cell.2012.11.039. 
28. Polo, J.M., Liu, S., Figueroa, M.E., Kulalert, W., Eminli, S., Tan, K.Y., Apostolou, E., Stadtfeld, M., Li, Y., Shioda, T., et al. (2010). Cell type of origin influences the molecular and functional properties of mouse induced pluripotent stem cells. Nat Biotechnol 28, 848-855. 10.1038/nbt.1667.

29. Rouaux, C., and Arlotta, P. (2013). Direct lineage reprogramming of post-mitotic callosal neurons into corticofugal neurons in vivo. Nat Cell Biol 15, 214-221. 10.1038/ncb2660.

30. Senis, E., Mosteiro, L., Wilkening, S., Wiedtke, E., Nowrouzi, A., Afzal, S., Fronza, R., Landerer, H., Abad, M., Niopek, D., et al. (2018). AAVvector-mediated in vivo reprogramming into pluripotency. Nat Commun 9, 2651. 10.1038/s41467-018-05059-x.

31. Seo, J.H., Lee, M.Y., Yu, J.H., Kim, M.S., Song, M., Seo, C.H., Kim, H.H., and Cho, S.R. (2016). In Situ Pluripotency Factor Expression Promotes Functional Recovery From Cerebral Ischemia. Mol Ther 24, 1538-1549.

10.1038/mt.2016.124.

32. Shapiro, S.D., Ranjan, A.K., Kawase, Y., Cheng, R.K., Kara, R.J., Bhattacharya, R., GuzmanMartinez, G., Sanz, J., Garcia, M.J., and Chaudhry, H.W. (2014). Cyclin A2 induces cardiac regeneration after myocardial infarction through cytokinesis of adult cardiomyocytes. Sci Transl Med 6, 224 ra227. 10.1126/scitranslmed.3007668.

33. Stadtfeld, M., Maherali, N., Borkent, M., and Hochedlinger, K. (2010). A reprogrammable mouse strain from gene-targeted embryonic stem cells. Nat Methods 7, 53-55. 10.1038/nmeth.1409.

34. Tan, K.Y., Eminli, S., Hettmer, S., Hochedlinger, K., and Wagers, A.J. (2011). Efficient generation of iPS cells from skeletal muscle stem cells. PLoS One 6, e26406. 10.1371/journal.pone.0026406.

35. Wang, C., Rabadan Ros, R., Martinez-Redondo, P., Ma, Z., Shi, L., Xue, Y., Guillen-Guillen, I., Huang, L., Hishida, T., Liao, H.-K., et al. (2021). In vivo partial reprogramming of myofibers promotes muscle regeneration by remodeling the stem cell niche. Nature Communications 12, 3094. 10.1038/s41467-021-23353-z.

36. Wesselschmidt, R.L. (2011). The teratoma assay: an in vivo assessment of pluripotency. Methods in molecular biology (Clifton, N.J.) 767, 231-241. 10.1007/978-1-61779-201-4_17.

37. Yilmazer, A., de Lazaro, I., Bussy, C., and Kostarelos, K. (2013). In vivo cell reprogramming towards pluripotency by virus-free overexpression of defined factors. PLoS One 8, e54754.

10.1371/journal.pone.0054754. 


\section{SUPPLEMENTARY FIGURES AND TABLES}

Figure S1. OKSM expression and pluripotency induction requires Myh6-specific Cre recombination, doxycycline administration and presence of the OKSM cassette.

Figure S2. OKSM expression in response to doxycycline (dose and time).

Figure S3. Changes in gene expression of TET enzymes upon doxycycline administration.

Figure S4. TEM of cardiac ventricle tissue of reprogrammable mice upon doxycycline administration.

Figure S5. Gene expression changes in atrial tissue upon doxycycline administration.

Figure S6. TEM of cardiac atrial tissue of reprogrammable mice upon doxycycline administration.

Figure S7. Gene expression in cardiac teratomas and ventricular myocardium.

Supplementary Table 1. Antibodies used in FACS studies.

Supplementary Table 2. List of primer sequences used in RT-qPCR studies in this work.

Supplementary Table 3. Antibodies used for immunohistochemistry and immunofluorescence in this study. 